\title{
A Closer Look at Power-Law Scaling Applied to Sea Surface Temperature from Scripps Pier Using Empirical Mode Decomposition
}

\author{
LAURENCE C. BREAKER ${ }^{\mathrm{a}, \mathrm{b}}$ AND DUSTIN CARROLL ${ }^{\mathrm{a}}$ \\ ${ }^{a}$ Moss Landing Marine Laboratories, Moss Landing, California \\ ${ }^{\mathrm{b}}$ University of Delaware, Newark, Delaware
}

(Manuscript received 8 August 2020, in final form 4 December 2020)

\begin{abstract}
The purpose of this study is to extract more information about the scaling exponents we obtain from sea surface temperature (SST) because their information content is limited to a single value. We examine the application of empirical mode decomposition (EMD) to power-law scaling using SST from Scripps Pier, California. The daily observations we employ extend from 1920 to 2009 , a period of 90 years. The annual cycle and the long-term trend were first removed. The decomposition produced a total of 15 modes. The scaling exponents were then calculated separately for each mode from the EMD. We have examined the distribution of scaling exponents with respect to the ensemble, and then with respect to the individual modes for the oceanic processes that we may infer from them. The first three modes are antipersistent and contain about one-quarter of the total variance. The pattern of modes that was obtained is continuous and relatively smooth beyond mode 3 with increasing values up to mode 8 and generally decreasing values thereafter. The pattern exhibits intramodal correlation, as expected, and intermodal correlation as well. Intermodal correlation is likely due, for the most part, to longrange persistence. The annual cycle in SST at Scripps Pier is a dominant feature in the record and contains almost $70 \%$ of the variance. A method for removing the annual cycle that is not based on removing the mean value is introduced and is recommended for future use.
\end{abstract}

KEYWORDS: Empirical orthogonal functions; Time series; Annual variations; Anomalies; Climate variability

\section{Introduction}

Power-law scaling is becoming of greater interest in the field of oceanography (e.g., Monetti et al. 2003; Luo et al. 2015; Breaker and Carroll 2019) and is frequently used in the atmospheric sciences (Tsonis et al. 1999; Fraedrich and Blender 2003; Vyushin et al. 2009). In applying power-law scaling a single scaling exponent is derived that relates the magnitudes of the fluctuations in the data to the scales they represent. Usually this exponent is similar or identical to the well-known Hurst exponent (Hurst 1951). Since the data usually take the form of time series the scales obtained are temporal. Powerlaw scaling provides a basis for describing or quantifying the relationship between the scales encompassed by the data. The scaling exponents that are obtained allow us to estimate longrange correlation (LRC) or the long-range persistence (LRP) of the system in question. A number of methods are used to estimate these exponents and four of the most popular methods are described in Witt and Malamud (2013). Detrended fluctuation analysis (DFA) is one of the methods that they evaluated, and we use it in this study (e.g., Kantelhardt et al. 2001; Kantelhardt et al. 2002; Ihlen 2012).

Although the magnitude of the scaling exponent provides information on the degree of LRC or LRP in the data and whether nonstationarity may be an issue, according to Vyushin et al. (2009), it has no simple established physical interpretation. We concur with Vyushin et al. (2009) on this question but note that the fluctuation function from which the scaling exponent is derived often provides additional information on the

Corresponding author: Larry Breaker, lbreaker@mlml.calstate.edu relationship between the scales involved (e.g., Tsonis et al. 2000). In concurrence with Vyushin et al. (2009), our primary objective is to investigate the scaling process in greater detail using empirical mode decomposition (EMD) to determine the contributions by mode to the scaling exponents we calculate. In this way we can observe which modes make the greatest contribution to our scaling results and infer, when possible, the physical processes that contribute to them.

\section{The data}

Daily observations of sea surface temperature (SST) have been acquired at Scripps Pier since 1916 in La Jolla, California, just north of San Diego. The observations we have chosen for analysis extend from 1 January 1920 to 31 December 2009, a period of 90 years. The end date was chosen for convenience in displaying the data since later in the study we employ only the first 89.78 years. The reason for this slight discrepancy will become clear in section 3d. For more information about the data from Scripps Pier see Checkley and Lindegren (2014).

The monthly-averaged observations of SST from Scripps Pier are displayed as a two-way layout showing variability on scales from intraseasonal ( $y$ axis) to interdecadal ( $x$ axis) (Fig. 1). Both intraseasonal and interdecadal warmings have been observed since the mid-1970s. In this regard there is a significant long-term warming trend that will be removed during the detrending process. We should also expect this record to reflect significant continental influence because of its close proximity to the coast (Yashayaev and Zveryaev 2001).

The primary reasons for selecting the record at Scripps Pier are its length and completeness. The record now exceeds 100 years (although we only use $\sim 90$ years). Also, because the annual cycle 


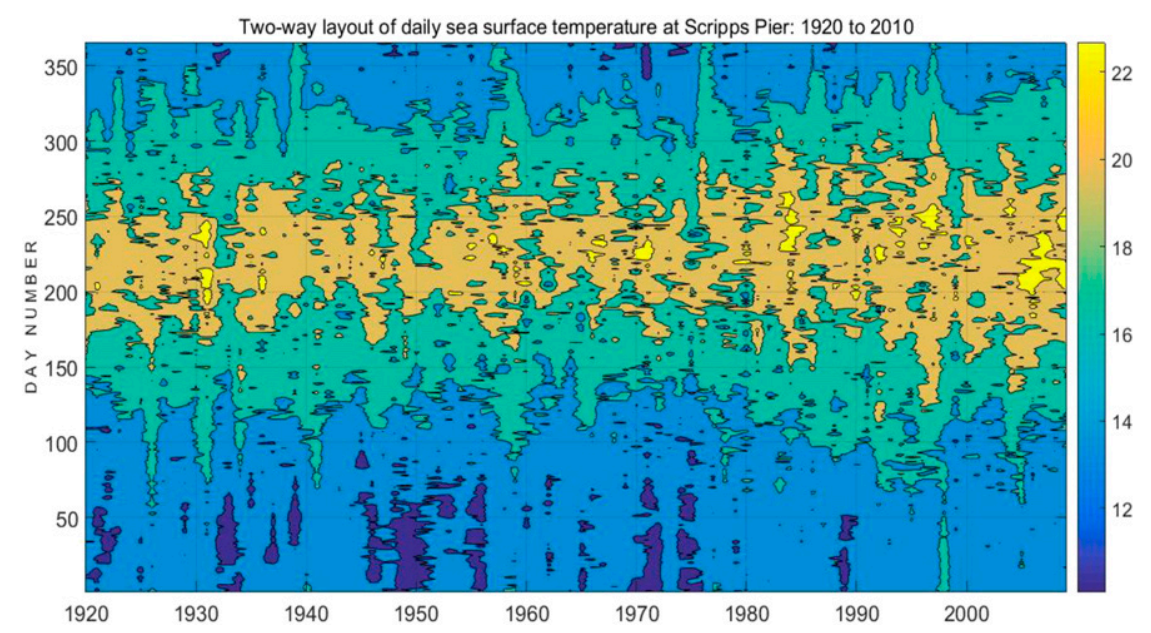

FIG. 1. Two-way layout of daily SST from Scripps Pier for the period from 1920 to 2009.

contains approximately $70 \%$ of the total variance it is more difficult to remove its influence, a step that is required in the detrending procedures we employ. Thus, removal of the annual cycle has also given us the opportunity to demonstrate the ability of a new method to effectively remove it from the data (see the appendix).

\section{Theory and methods}

\section{a. Theory}

The time-dependent structure of a linear random process can be described by the autocorrelation function (ACF; e.g., Jenkins and Watts 1968). The ACF expresses the relationship between $x(t)$ and lagged versions of the same time series, $x(t+$ $k$ ), where $k$ represents the time separation or lag between $x(t)$ and $x(t+k)$. The ACF can be expressed as

$$
C(k)=1 / N \sum_{t=1}^{N-k}[x(t)-\bar{x}][x(t+k)-\bar{x}],
$$

where $N$ is the record length, and $\bar{x}$ is the mean value of $x(t)$ for $t=1,2, \ldots, N$. Two different classes of ACF can be distinguished, following Maraun et al. (2004). The first class is characterized by short-range correlations where $C(k) \propto e^{-k / \tau}$ for $k \rightarrow \infty$, and $\tau$ corresponds to a characteristic time scale for the process. For such processes, $C(k)$ decays exponentially and so may become negligible even for relatively small values of $k$. Such processes correspond to short-term persistence. In the second case, there are processes that display ACFs that decay at much slower rates. Here the decay process is characterized by algebraically decaying correlations with the ACF decreasing as a power law for large values of $k$. Thus, $C(k) \propto k^{-\gamma}$ for $0<\gamma<1$, where $\gamma$ is the correlation exponent. Such processes are often said to display long-range correlation, dependence, or persistence. LRP is characterized by scale invariance (or at least to an approximation thereof), a property that can be quantified by calculating $\gamma$, which describes the relationship between time scales (Abry et al. 1998).

As indicated above, we employ DFA to estimate the magnitude of the fluctuations in SST as a function of time scale. We then obtain a fluctuation function, $F(k)$ as a function of $k$. If $F(k)$ is found to increase according to a power law, then this relationship can be expressed as $F(k) \propto k^{\alpha}$ for relatively large values of $k$ where $\alpha$ is referred to as the fluctuation exponent. It has been shown for large values of $k$ that $\alpha$ and the correlation exponent, $\gamma$, are related according to $\alpha=1-(\gamma / 2)$ (Taqqu et al. 1995). Thus, the results of DFA are directly related to the correlation exponent in the ACF. When we employ random noise in calculating $\alpha$ we obtain a value of 0.5 (or $\gamma=1.0$ ), which indicates the absence of long-range correlation in the data. When the value of $\alpha$ exceeds 0.5 , then long-range persistence is taken to be present. For values of the scaling exponent that exceed 1.0, nonstationarity as well as strong persistence may occur. As pointed out by Vyushin et al. (2009), beyond the degree of persistence that is indicated by the value of the scaling exponent per se, the power-law model has no established physical interpretation although the fluctuation function itself often provides additional information.

It is important to note that LRC in the data should not include nonstationary behavior (Chen et al. 2002; Hu et al. 2001). As a result, the influence of periodicities or cyclic behavior such as the annual cycle must be removed before realistic estimates of LRC can be obtained. The same is true for long-term trends in the data that must likewise be removed.

\section{b. Empirical mode decomposition}

In more detail, EMD is a method for decomposing a time series into a sequence of empirically orthogonal intrinsic mode function components (IMFs) and a residual (Huang et al. 1998). EMD is data adaptive, and well suited for the analysis of nonstationary and nonlinear time series. In EMD, the number of modes is determined by the data. Each IMF represents a mode of oscillation with time-dependent amplitude and frequencies that lie within a specific band of frequencies, the center of which defines the mean period of that mode. The process of extracting the individual modes or essential scales from the data is called sifting and is performed many times to produce a single IMF. The IMFs are often physically meaningful because the characteristic scales in each case are 
determined by the data. Stated more explicitly, because the modes are orthogonal each IMF captures a single aspect of the dynamics of the system.

In the process of sifting, local maxima and minima are identified in the record and envelopes are formed by fitting cubic splines to the extreme values. The differences between the envelope and the mean provide an estimate of the first IMF. Once the first IMF (imf1) has been obtained, it is subtracted from the original data $x(t)$, producing a set of residuals $r_{1}$ that can be expressed as

$$
r_{1}=x(t)-\operatorname{imf} 1
$$

The residuals $r_{1}$ are then subjected to the same process, yielding the second IMF (imf2) as

$$
r_{2}=r_{1}-\operatorname{imf} 2,
$$

and so on, until a final residual is obtained that often corresponds to the long-term trend.

One problem in the application of EMD was that mode mixing often occurred when a time series included intermittently occurring signals with widely separated time scales. To address this problem, EMD now includes a noise-assisted component in its calculation. Wu and Huang (2008) developed a technique now called "ensemble EMD," or EEMD, that defines the true IMF as the mean of an ensemble of IMFs, and, in fact, preserves the physical uniqueness of the decomposition. An ensemble member consists of the signal plus white noise of finite amplitude. The magnitude of the white noise that should be added is given by the ratio of the standard deviation of the first IMF to the standard deviation of the data itself and is called $N_{\text {std }}$. The number of realizations or ensemble size that is generated may be several hundred or more. Thus, in EEMD there are two free parameters that must be specified, the level of white noise to be added and the ensemble size. The ensemble size that we have used in this study is 300 [for more information on EMD, see Huang et al. (1998) and Huang (2005a,b); for more information on EEMD, see $\mathrm{Wu}$ and Huang (2008)].

Following Capparelli et al. (2011), which is based on EEMD, our SST time series $\mathrm{T}(t)$ can be decomposed into a finite number of IMFs according to

$$
T(t)=\sum_{j=0}^{n-1} \varphi_{j}(t)+r_{n}(t),
$$

where $\varphi_{j}(t)$ represents the $j$ th mode with its own time scale and a zero mean. Each mode may be subject to various sources of variability within its band-limited framework including amplitude and phase modulation. The last term in Eq. (4), $r_{n}(t)$, is what remains after all of the lower modes have been removed from the original data and so is often referred to as the residual. Thus, it often corresponds to any long-term trend that may be contained in the data. This method of decomposition, based on the work of Huang et al. (1998) and more recently stated by Capparelli et al. (2011), is local, complete, and orthogonal.

Although Capparelli et al. (2011) have successfully used this approach to separate and remove the annual cycle and the long-term trend from a record of surface air temperature we find that problems can arise in removing the annual cycle when it is not confined to a single mode. If more than one mode is removed in order to remove the annual cycle, then there may be an unnecessary loss of information in the process. As a result, we have used a narrowband analysis performed in the frequency domain to remove the annual cycle (see the appendix).

\section{c. Detrended fluctuation analysis}

DFA is a method whose first step is to remove any longterm trends in the data. It is flexible in that the degree of detrending can be adjusted and so is chosen by the user. Once the detrending is performed then a scale analysis is conducted where we calculate the magnitude of the variability as a function of the time scale in a manner similar to calculating the variance. If we use monthly data, for example, we might examine time scales from several months to several years depending on the record length. At this point we can calculate the magnitude of the variability versus time scale, and if we plot the results in log-log coordinates, the scaling exponent corresponds to the slope of the corresponding plot.

In greater detail, DFA is a method for estimating the scaling behavior of noisy data in the presence of nonstationarities (Peng et al. 1994). Following Kantelhardt et al. (2001), the method is composed of five steps. The first step can be expressed as

$$
y(t)=\sum_{k=1}^{t}[x(k)-\bar{x}],
$$

where the same symbols were used earlier and $y(t)$ is the cumulative sum of $x(t)$. This step is often referred to as creating the profile or aggregate. This step is necessary if the time series possesses significant noise-like structure, which is true for most geophysical time series (Ihlen 2012).

In the second step, $y(t)$ is divided into $N_{s}$ nonoverlapping segments $N / s$ of equal length $s$. If $N$ is not a multiple of $s$, there will be a small portion of the record that will not be included in the calculation. One way to make good use of the unused segment is to reverse the data and then proceed with the same calculation a second time. This has the advantage of making full use of the data plus obtaining a second estimate of the scaling exponent, albeit one that is far from being independent of the first.

In the third step, the local trend $v$ is calculated for each segment based on a least squares fit to the data. The basis function that is chosen to provide the fit can be linear, quadratic, cubic, a higher-order polynomial, or even EMD as Qian et al. (2009) have done. To simplify the notation, we refer to linear detrending as DFA1, quadratic detrending as DFA2, cubic detrending as DFA3, and so forth. We follow this notation throughout.

In the fourth step, the variance $F_{s}^{2}$ for each of the $N_{s}$ segments is calculated as

$$
F_{s}^{2}(v)=\frac{1}{s} \sum_{i=1}^{s}\left\{y[(v-1) s+i]-p_{v}(i)\right\}^{2},
$$


EEMD for original SST from Scripps Pier: 1920 - 2010

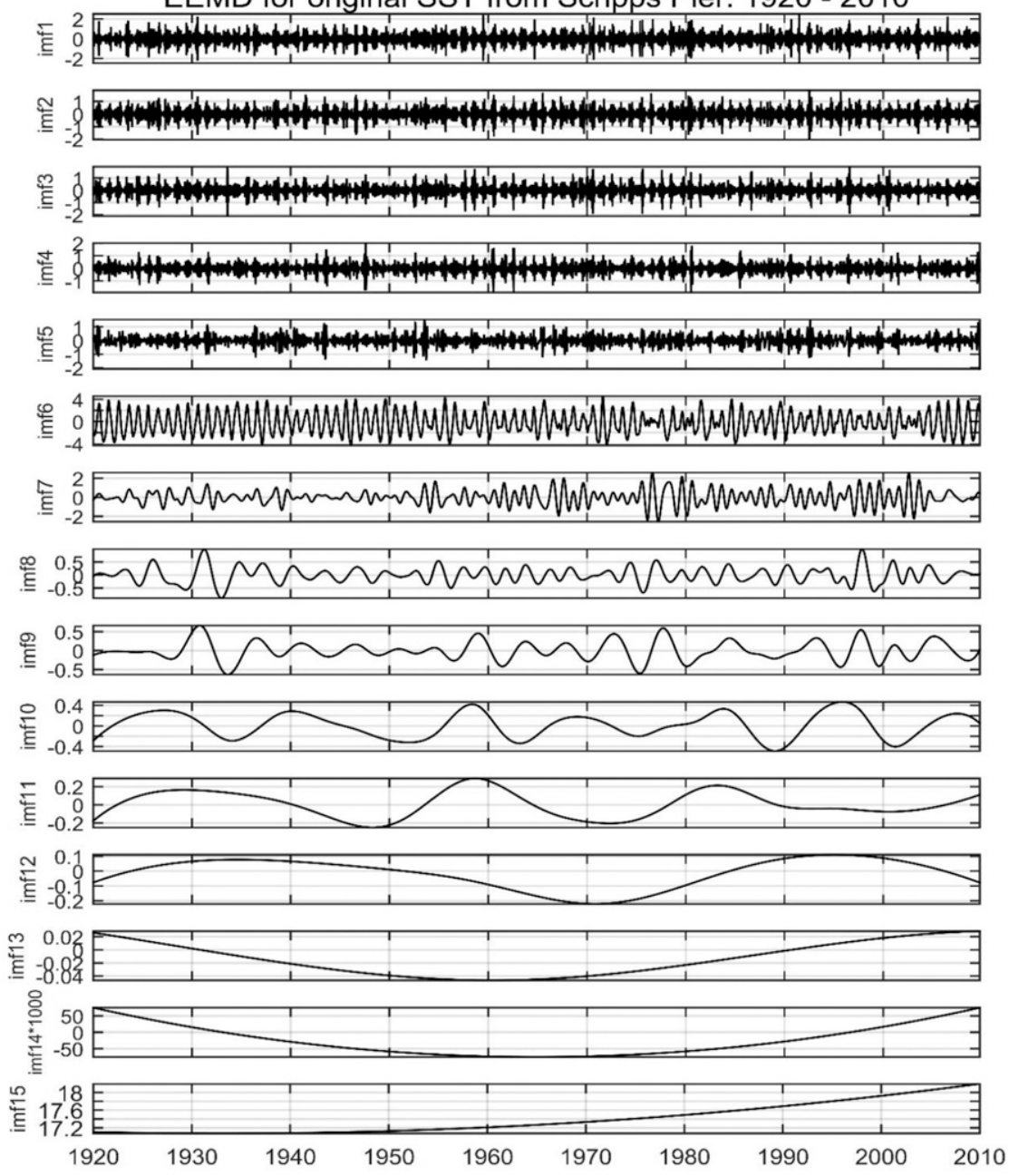

FIG. 2. Ensemble empirical mode decomposition of daily SST from Scripps Pier from 1920 to 2010

for each segment $v$, where $v=1, \ldots, N_{s}$, and $p_{v}(i)$ is the fitting polynomial for segment $v$.

The fifth step is to average over all of the segments and then take the square root of the mean squared values to obtain what is referred to as the fluctuation function for each value of $s$, according to

$$
F_{s}=\frac{1}{N_{s}} \sum_{v=1}^{N s} F_{s}^{2}(v)^{1 / 2} .
$$

If the data follow a power law resulting from long-range correlation, the fluctuation function $F_{s}$ increases according to

$$
F_{s} \propto s^{H}
$$

where $H$ is the final scaling exponent (often referred to as simply the scaling exponent elsewhere in the text, and in other references often referred to as the Hurst exponent). To obtain this value we plot $F_{s}$ versus $s$ in $\log$-log coordinates to the base 2 (in our case) and then determine the slope of the line from a least squares linear fit to the data. Although $H$ is the end product of these calculations, $F_{s}$ itself often provides additional information that cannot be obtained from $H$ alone.

\section{d. Scaling considerations}

Our goal is to decompose the time series of SST from Scripps Pier into a sequence of IMFs or modes using EEMD and then to determine the contribution of each mode to the scaling exponent we obtain. To accomplish this goal, we must first select the scales that we will use to calculate the scaling exponent. We employ a period of 32768 days, which corresponds to 89.78 years, close to the 90 years we used earlier to plot the data and apply EEMD. The scaling we have adopted is the following: [16 3264 1282565121024204840968192 ], where the scales are given in days and span a range of 10 (from 16 days to 22.4 years). We start on 1 January 1920 and end on 12 October 2009, just short of 90 years. Of interest here are the choices of the smallest and largest scales. The choice of 16 days is reasonable since it is slightly longer than the estimated correlation time scales for SST along the coast of California for observations acquired daily 


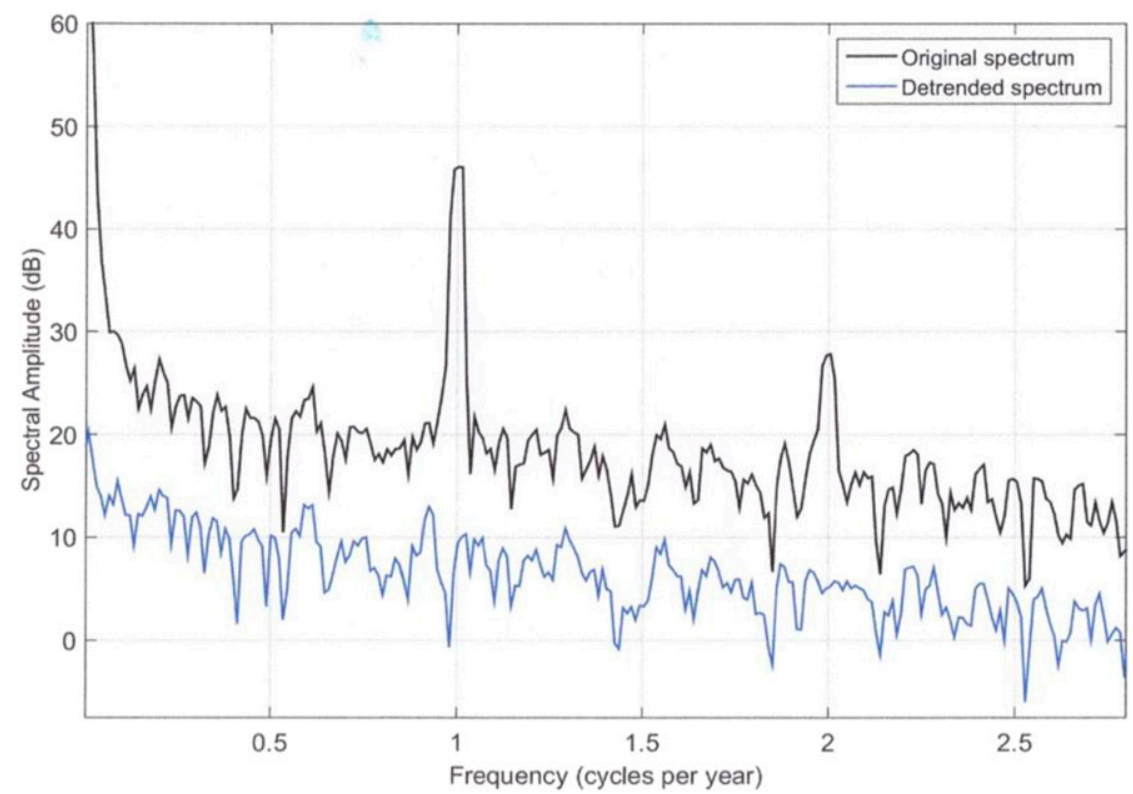

FIG. 3. MTM power spectra for SST from Scripps Pier for the period from 1920 to 2010 . The original data are shown in black, and the detrended data with the long-term trend and annual cycle removed are in blue. The plots are offset by $10 \mathrm{~dB}$ to improve readability.

(e.g., Breaker et al. 2011). The choice of the longest time scale is a different matter. It depends on how many subsamples within the analysis period are considered sufficient to provide a reliable estimate of $F_{s}$ when $s$ is equal to the maximum scale. From the literature the minimum number of subsamples preferably should not be less than 4 (Fraedrich and Blender 2003; Bunde et al. 2004). Since our record is of sufficient length, the numbers that we have chosen make the issue of adequate sampling a simple matter since 32768/8192 $=4$. In this work the size or scale of the subsamples is usually chosen to increase as powers of 2 or 10 for the obvious reason that it facilitates plotting the results in logarithmic coordinates from which we obtain the scaling exponents.

\section{The results}

\section{a. The EEMDs}

The first step in our analysis has been to apply EEMD to the daily observations from Scripps Pier. The results of this decomposition are shown in Fig. 2. The data were decomposed into 15 modes. The highest mode, i.e., mode 15 , as stated earlier, usually contains the long-term trend although sometimes additional modes are included depending on how the longterm trend is defined. In this case, we consider mode 15 by itself to be the long-term trend. It is slightly curvilinear and indicates an overall increase in temperature of approximately $1.0^{\circ} \mathrm{C}$ over the period from 1920 to 2010 and carries approximately $1.5 \%$ of the total variance.

The long-term trend and the annual cycle both contribute to nonstationarity in the data and so must be removed before further calculations are performed (e.g., Hu et al. 2001; Chen et al. 2002). Based on the EEMD it is relatively straightforward to remove the long-term trend by simply subtracting the highest mode, that is, mode15, from the original data. This is possible because the modes, as indicated above, are independent (e.g., Huang et al. 1998). Although Malamud and Turcotte (1999) recommend removing only the linear contribution to the trend, we expect that any distortion to the spectrum that may occur will be found only at the lowest frequencies $(<0.1$ cycle per year).

The next step is to remove the annual cycle. We use a narrowband filter to remove the annual cycle, but, in the process, gaps are created where cycles existed that must be filled with proxy values. In following this course of action, we can produce a complete time series that is generally representative but does not contain the annual cycle, gaps, or a long-term trend. The details of how the annual cycle is removed are contained in the appendix. A comparison of the power spectrum of the original data and the detrended data is shown in Fig. 3.

Next, we apply EEMD once again but this time to the detrended data (Fig. 4). Modes 1-5 are generally similar, but starting with mode 6 they differ significantly. The annual cycle, clearly visible in mode 6 and, to a lesser extent, mode 7 of Fig. 2, is almost nonexistent in Fig. 4 (do note, however, that the scales generally differ between the two figures). From mode 6 on, the modal patterns have been altered significantly in the majority of cases. This should not be surprising since we have just removed $\sim 70 \%$ of the variance from the record. Although we removed the long-term trend initially a small positive trend still exists. Its appearance in the detrended spectrum (Fig. 3), however, is far less prominent.

Last, we calculate the variances by mode from the EEMDs for the original data and the detrended data. The results are 
EEMD for detrended SST from Scripps Pier: 1920 - 2010
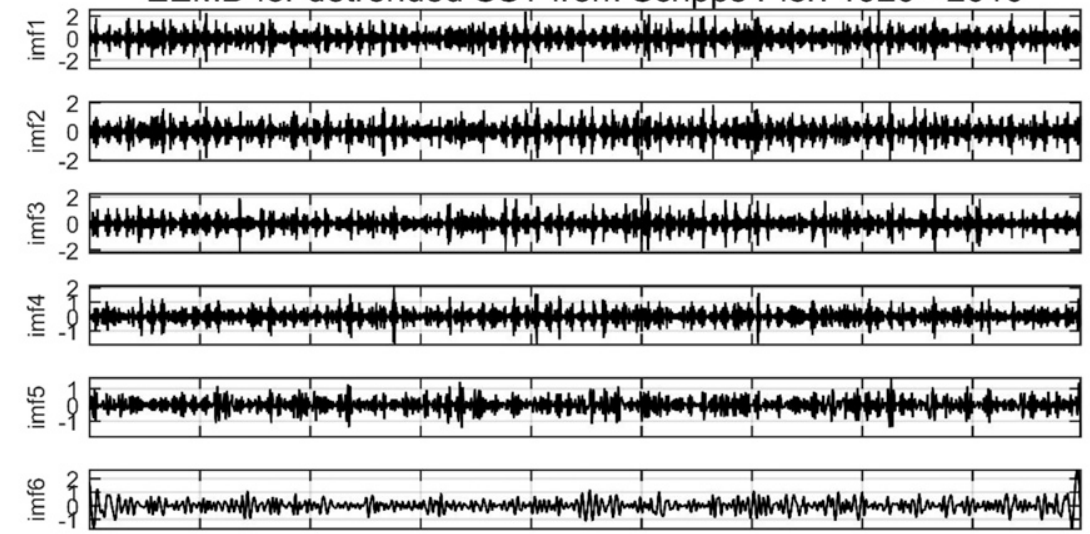

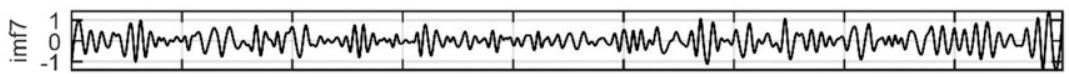
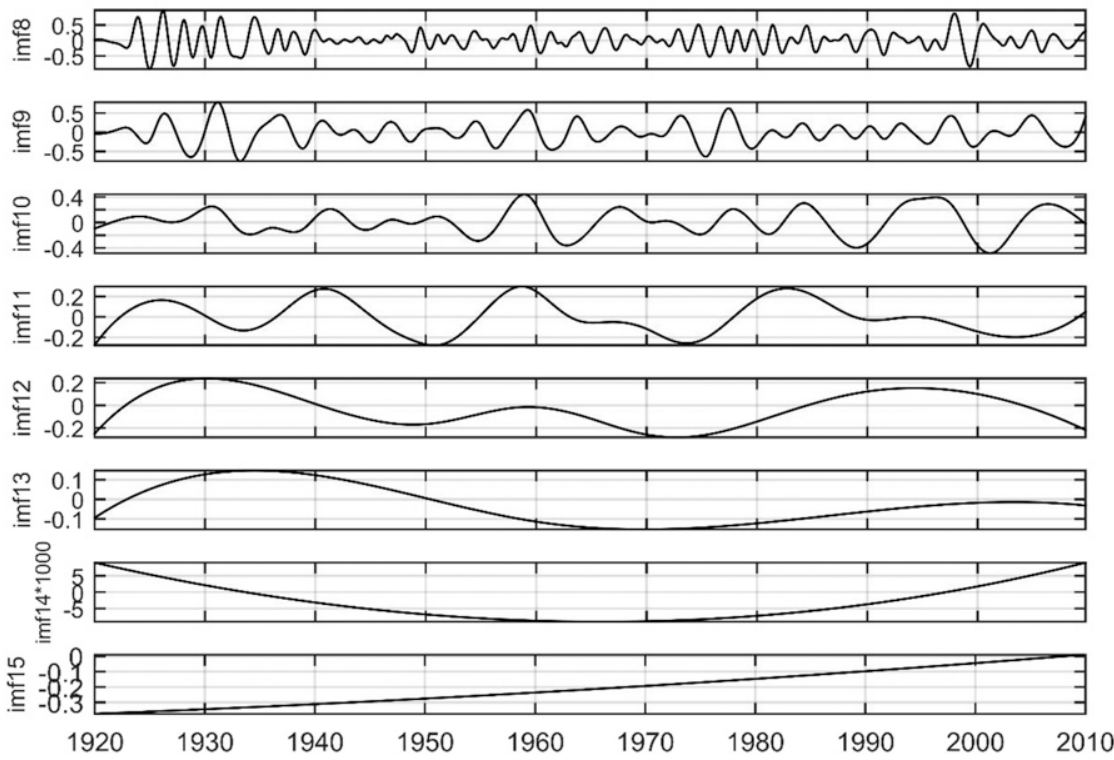

FIG. 4. EEMD for the same data shown in Fig. 2 except now the annual cycle and the long-term trend have been removed (i.e., the data have been detrended).

shown in Fig. 5 with the ordinate plotted in decibels. As we expect, the results are essentially identical until we reach mode 6 . The variance associated with the annual cycle is shared between modes 6 and 7 as indicated earlier in Fig. 2. After mode 7 the variances, both detrended and original, start to decrease significantly, a trend that continues until the last mode (i.e., mode 15) where the long-term trend makes a major contribution to the original data. The annual cycle serves as a changepoint or period with respect to the distribution of variances where the lower modes $(<$ mode 6$)$ are consistently higher and the higher modes ( $>$ mode 7$)$ are consistently lower. By the time we reach the last four modes the variances are relatively small and have little impact on the results with the exception of the long-term trend for the undetrended version. In this last case the variance is significantly higher by $5-10 \mathrm{~dB}$ for the undetrended version but the variance for the detrended version is $7-8 \mathrm{~dB}$ lower, showing the impact of removing the long-term trend.

\section{b. EEMD and the fluctuation functions}

As mentioned earlier, it is not a simple matter to extract information about the oceanic processes that may influence the scaling directly from the scaling exponent itself. As discussed in section 3, we apply DFA to calculate the scaling exponents using the detrended data. Specifically, we use DFA4 to apply additional detrending although this step is sometimes unnecessary. When we apply DFA4 to the detrended data in its entirety we obtain a value of 0.96 for the scaling exponent.

Although the scaling exponents themselves usually provide little information about the processes involved, by including the fluctuation functions that are used to derive the scaling exponents we often obtain additional information. 


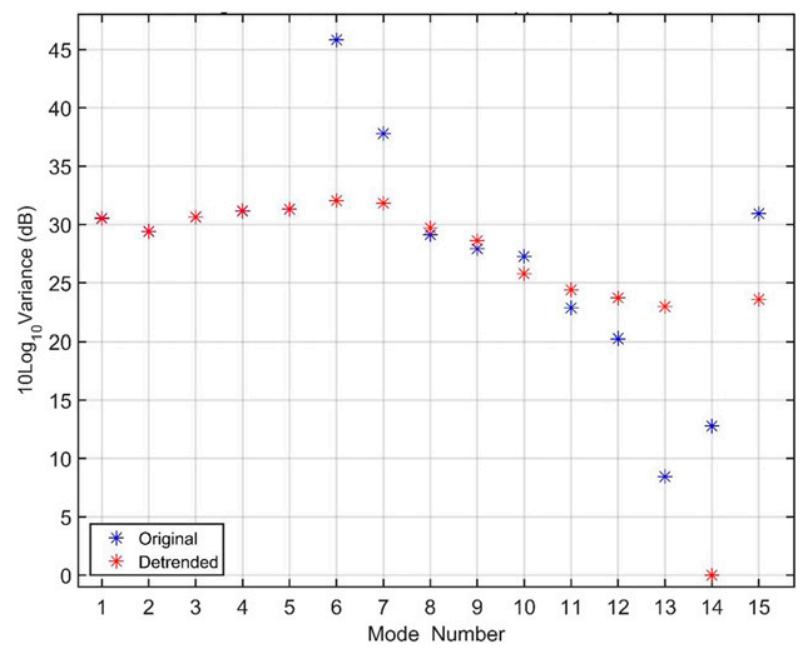

FIG. 5. Variances by mode for the original data in blue and the detrended data in red.

For example, when we plot the fluctuation functions per se, we see the number of scales that are employed at a glance. We can also observe the relationships between scales and the degree to which linearity or scale invariance is obeyed (or violated). Last, we may also observe interference or crossover problems associated with El Niño-Southern Oscillation (ENSO) that are often encountered at lower latitudes.

The fluctuation functions are plotted in Fig. 6 for each of the mode groupings starting with all 15 modes, followed by modes $1-14$, modes 1-13, and so on through the entire sequence until the calculation is applied to mode 1 only. This figure shows the results with each function offset from its neighbor by a constant value to avoid overlap. First, we note a departure from linearity or possibly a crossover at a scale of 10 that corresponds to 5.6 years and may represent the influence of ENSO, generally consistent with the results of Luo et al. (2015). This pattern persists only for the highest modes down to about modes 1-9 at which point the influence of ENSO appears to have been effectively removed. Thus, the nature of the departure changes to some degree when we include only the first eight and lower modes.

We note that modes 6 and 7 serve as proxies since the annual cycles have been removed and the gaps filled (see the appendix). Hence, it is difficult to attach any physical interpretation to them although they still represent a significant portion of the variance (Fig. 5). We also observe that scale invariance, which may serve as a useful approximation for the higher modes, no longer applies for the lower modes. As the modes are sequentially removed the variances at the longer scales are likewise removed reducing the values of $F(s)$ accordingly at those scales and thus decrease the slopes as well.

\section{c. EEMD and the scaling exponents per se}

To extract additional information from the scaling exponents we apply EEMD with a twist. In this case we calculate the scaling exponents separately for each mode from the EEMD (Fig. 7). Flandrin et al. (2004, 2005) used spectrum analysis in a

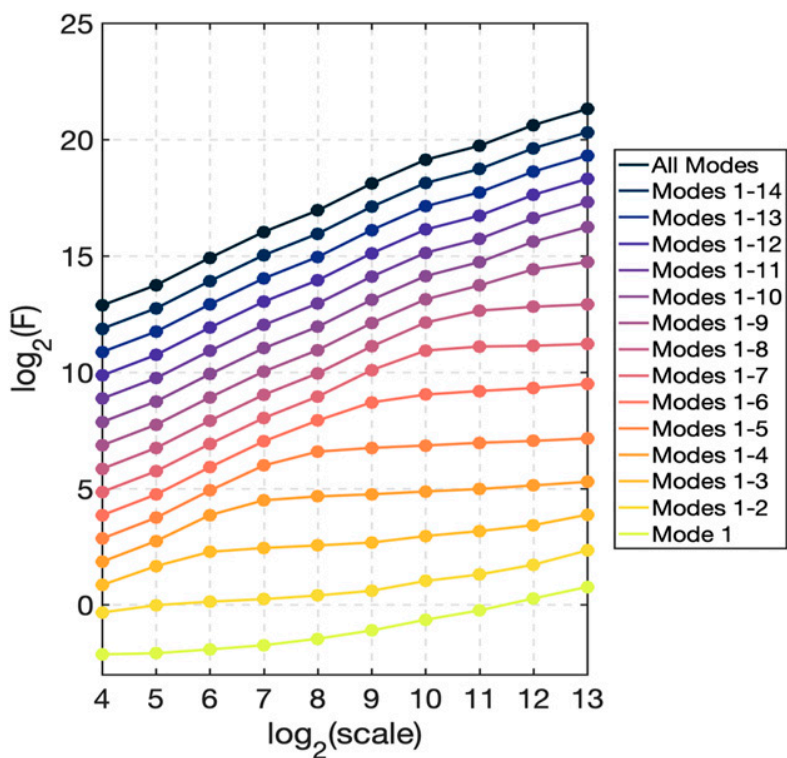

FIG. 6. Plots of the fluctuation function $F(s)$ vs the scale $s$ for the mode groupings shown in the legend. Each plot has been offset by a constant amount to avoid overlap.

similar way to observe how a complete spectrum is divided into IMFs using fractional Gaussian noise as the basis. First, we note that the amplitude of mode 14 is not consistent with its neighbors, a fact that is almost certainly due to its relatively small detrended variance.

Next, we note that after the second mode the individual values of the scaling exponents, that is, $H$, form a relatively smooth and continuous function although the trend following mode 8 changes from increasing to decreasing values. At variance with this pattern are the first three modes, which are notably less than 0.5 and thus antipersistent, indicating that adjacent values in the record are negatively correlated. The first three modes also contain approximately $26 \%$ of the total variance in the data, which is not an insignificant amount. As we have observed from at least the fourth mode, the pattern of values for $H$ are generally smooth and continuous and we expect a high degree of intramodal correlation, but intermodal correlation was not necessarily expected and yet is clearly evident. Even the proxies for modes 6 and 7 tend to follow the same pattern. Of note is the evidence for intermodal correlation, which does not appear until the third or fourth mode. This behavior is almost certainly related to LRP, and perhaps to a lesser degree, nonstationarity. We expect uniformity and smoothness to be characteristic of LRP and to exhibit a prevailing influence overall, whereas nonstationarity would be expected to exhibit greater variability between the modes if it played a greater role but that is not what we observe. Consistent with the increasing influence of LRP as the values of $H$ increase, we also observe that the patterns of ascending and descending $H$ have greater curvature (i.e., smaller radii of curvature) for the higher values, and lower curvature (i.e., larger radii of curvature) for the lower values. In addition, the symmetry of the scaling exponents with respect to the vertex at 


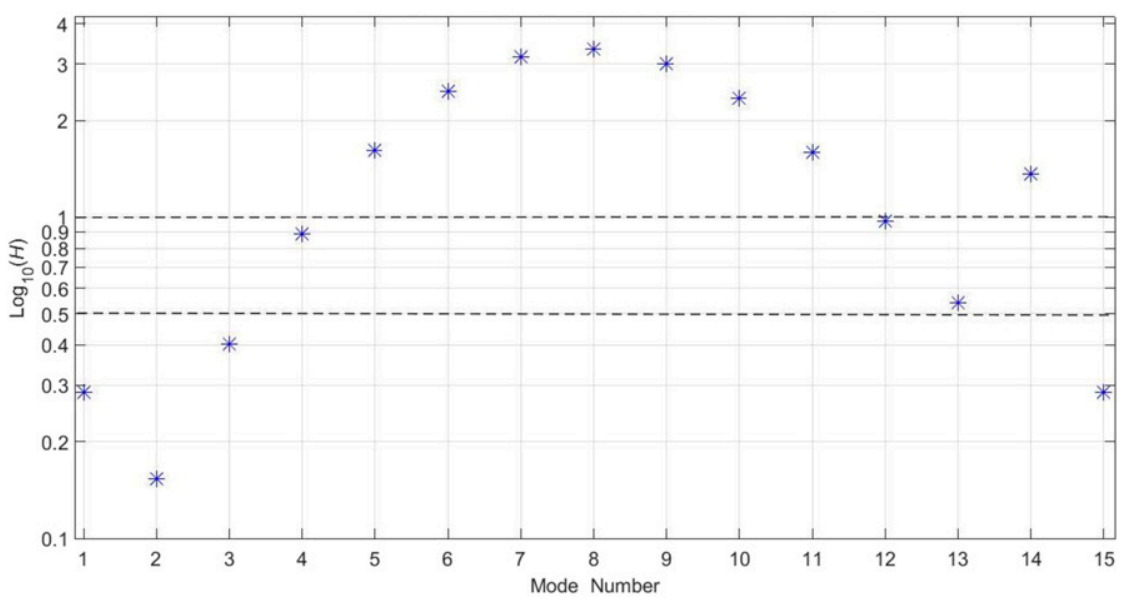

FIG. 7. The individual scaling exponents plotted on a logarithmic scale vs mode number, where $H$ represents the scaling exponent. The two horizontal dashed lines bracket the range of values that correspond to LRP. The scaling exponent for mode 14 is not reliable because the detrended variance is too small.

mode 8 provides further evidence that LRP strongly influences how the scaling exponents are distributed. Last, the correlative properties associated with LRP and perhaps nonstationarity would be expected to have time scales that exceed those associated with the individual modes.

Only three values, that is, modes 4,12 , and 13 , fall within the range from 0.5 to 1.0 where LRP per se occurs. From the fifth mode on, although we primarily observe the influence of LRP, it is possible that nonstationarity could have some impact. At values of $H$ that clearly exceed 1.0 it may be difficult to distinguish between the effects of nonstationarity and LRP.

The positive trend for $H$ from the third or fourth mode up through mode 8 is clear. The detrended variances associated with these modes are relatively high and vary only slightly (Fig. 5). By mode 8, however, the variances have started to decrease and they decrease steadily and significantly until the last mode, i.e., mode 15 . As a result, the ability of modes 8 through 14 to contribute to the overall scaling is strongly reduced. In fact, modes $1-5$ alone carry $48.8 \%$ of the variance, or almost one-half of the total variance in the detrended data.

The overall value for $H$ was 0.96 for the detrended data but only three values of the individual scaling exponents fell between 0.5 and 1.0 while at least 11 values did not. Although antipersistence, as exhibited by the first three scaling exponents, acts to lower the scaling exponents, nonstationary behavior, if present, could contribute to the values that exceed 1.0. For values of $H$ that exceed 1.0, processes that are strongly stationary contribute to LRP as well as nonstationarity. According to Malamud and Turcotte (1999), on the scale of increasing persistence, 1.0 represents a natural crossover between weak and strong persistence.

Such a wide range of scaling exponents was not anticipated and could raise the question as to the representativeness of a single value. Thus, it could be argued that a single value for all of the modes may oversimplify the problem.

The time scales associated with each mode are shown in Table 1 and were obtained from spectral analysis using the maximum taper method (Thomson 1982) with a timebandwidth product of 3 , and peak-to-peak time separations for the longest time scales. It is difficult to relate the statistical properties of the first three modes with the physical processes that are known to occur at these time scales (i.e., from several days to roughly 20 days). Also, as stated earlier, the sum of the variances in the first 3 modes is at least $25 \%$, providing a measure of their relative importance. We know that antipersistence as shown by the first three modes is characterized by a lack of persistence and thus would be expected to exhibit a relatively high degree of random behavior. This, in turn, is consistent with primarily wind-driven variability that has a relatively large random component. Wind forcing is responsible for the generation of surface gravity waves, small-scale currents and vigorous vertical mixing within the upper layers of the ocean. We should expect to observe the influence of wind forcing on time scales of up to several months or more (e.g., Monin et al. 1972; Ackerman 1981; Jones and Toba 2001; Wells 2012; Watson 2014).

Mode 4 corresponds to LRP per se and mode 5 indicates LRP along with possible influence from nonstationary behavior (with time scales of approximately 40 and 90 days, respectively). As the mode number increases from 4 to 6 , we should observe the emergence of ocean features than can be uniquely identified off the California coast such as indicators of coastal upwelling including ocean fronts, meanders, jets, offshore Ekman transport, cyclonic and anticyclonic eddies, and offshore upwelling. These features may be expected to persist on monthly to seasonal time scales and longer. Modes 6 and 7 are strongly influenced by the annual cycle and were initially removed and at this point simply serve as proxies. Mode 8 has the highest value of $H(3+)$ and so a nonstationary contribution might not be unexpected. This mode falls between the annual cycle and the El Niño/ENSO phenomena (i.e., from $\sim 1$ to $4-$ 5 years) and, as a result, contributes to biannual variability. El Niño/ENSO occupies at least modes 9 and 10, with a transition that occurs from modes 9 and 10 to modes 11 and 12, as the Pacific decadal oscillation (PDO) emerges at longer time 
TABLE 1. Results from spectral analysis and peak-to-peak time separation.

\begin{tabular}{cc}
\hline \hline Mode & $\begin{array}{c}\text { Center frequency in cycles per year } \\
\text { (period in days or years) }\end{array}$ \\
\hline 1 & $100-108(3.5$ days $)$ \\
2 & $38.5(9.5$ days $)$ \\
3 & $18.3(20.0$ days $)$ \\
4 & $8.9(41.0$ days $)$ \\
5 & $4.0(91.3$ days $)$ \\
6 & $1.9(192.0$ days $)$ \\
7 & $0.97(376.3$ days) \\
8 & $0.50(730$ days $=2$ years $)$ \\
9 & $0.21(4.76$ years $)$ \\
10 & $0.10(10$ years $)$ \\
11 & $0.04(25$ years $)$ \\
12 & $0.0166(60$ years) \\
13 & 0.0091 (110 years) \\
14 & Not resolved \\
15 & Trend removed \\
\hline
\end{tabular}

scales. Consistent with this transition, modes 11 and 12 have time scales of approximately 25 and 60 years, respectively. Mode 13 has a time scale of roughly 110 years and so could be related to solar variability. The time scales associated with modes 11, 12 and 13 were obtained using peak-to-peak separation times because of the obvious limitations of spectral analysis at this point.

In closing, we have attempted to reconcile the statistical properties of the lower modes, in particular, with the known physical processes that occur at similar time scales. As Huang (2005a) has indicated, the individual IMFs are often physically meaningful since the characteristic scales that occur are defined by the observations per se and thus provide additional support for this reconciliation.

\section{Discussion}

The work presented here in some respects is reminiscent of the work reported by Flandrin et al. (2004, 2005). Their approach was to apply EEMD to a known or specified source of noise or scaling process to better understand how a complete spectrum can be divided into IMFs. Their results indicated that in the case of fractional Gaussian noise (e.g., Mandelbrot and van Ness 1968) the properties of an equivalent filter bank structure were revealed, and except for the first mode, that all of the higher modes can be described as a set of bandpass filters or dyadic filter bank with a constant $Q$ (quality factor).

Our work, although based on modal decompositions from EEMD, departs significantly from Flandrin et al. (2004, 2005) since the basis for it has been to employ an observed time series where problems such as detrending, nonstationarity, and scale variance must be addressed.

\section{Conclusions}

Our primary goals have been to examine the distribution of scaling exponents, i.e., the values of $H$ by mode based on an observed time series to shed light on intermodal variability and correlation, to examine the relationships between the individual modes and, finally, to identify and prioritize the various oceanic and atmospheric processes that contribute to the scaling process.

The fluctuation functions from which the scaling exponents were derived permit us to routinely observe not only the number of time scales that are employed, but of greater importance, the relationships between the scales and the degree to which linearity or scale invariance has been obeyed. The fluctuation functions also allow us to observe interference or crossover problems associated with ENSO that are often encountered in SST at lower latitudes.

The scaling exponents have been calculated for each mode of the EEMD. The resulting pattern beyond mode 3 is continuous and relatively smooth with increasing values up to mode 8 and decreasing values, with one exception, thereafter. The pattern exhibits not only intramodal correlation, as expected, but also intermodal correlation. Intermodal correlation that is not apparent in the first two or three modes is almost certainly related to LRP, and possibly to a lesser extent, nonstationarity. These properties arise at least for the higher modes with the higher scaling exponents, where the time scales associated with LRP and possibly nonstationarity would be expected to exceed those associated with the individual modes. The importance of LRP was also clearly suggested by the distribution of the scaling exponents. The patterns of curvature and the symmetry shown by these exponents are consistent with what one might expect from the influence of LRP. In addition, the mostly decreasing values of $H$ that occur after mode 8 may be primarily due to the significantly lower variances for the higher modes with almost $50 \%$ of the total variance contained in the first five modes.

Last, the annual cycle in SST at Scripps Pier contains almost $70 \%$ of the variance in the record. A new method for removing the annual cycle and filling the resulting spectral gaps has been developed and has been applied to the data from Scripps Pier with apparent success. Although we recommend the procedures that were employed for general use they could be automated or at least partially automated for easier application.

Acknowledgments. The authors thank the Shore Stations Program at the Scripps Institution of Oceanography for the data that have been used throughout this study. We also acknowledge the very helpful comments from two anonymous reviewers.

\section{APPENDIX}

\section{Removing the Annual Cycle}

The annual cycle cannot be adequately removed using the standard definition of the temperature anomaly that is obtained by simply removing the mean annual cycle from a series of observations. This problem is well recognized both for surface air temperature (e.g., Capparelli et al. 2011) and SST (e.g., Breaker 2006). 

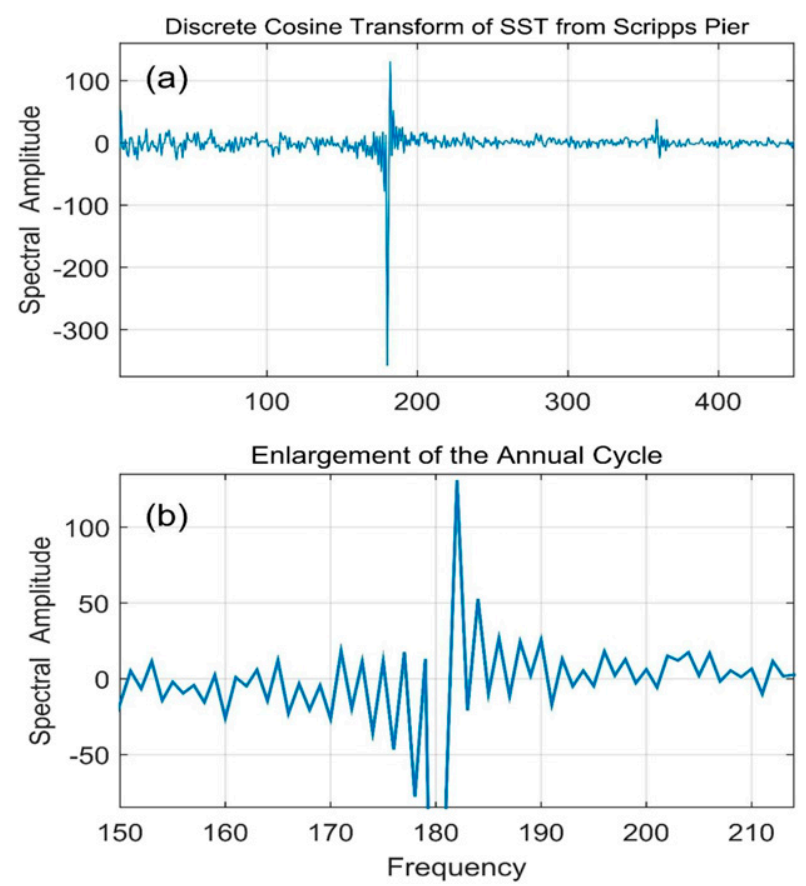

FIG. A1. (a) The discrete cosine transform of the daily SSTs from Scripps Pier in units of relative frequency. (b) An enlarged version of (a) centered on the annual cycle, again in units of relative frequency.

A method for removing the annual cycle and providing a proxy in its absence was presented by Breaker and Carroll (2019). The method involved several steps. First, the annual cycle and its first harmonic, often called the semiannual cycle, were removed using singular spectrum analysis (e.g., Golyandina et al. 2001). In this capacity, singular spectrum analysis (SSA) served as a tunable band-rejection filter and, although serving well as a filter, it left significant gaps in the spectrum at the two primary harmonic frequencies (i.e., 1 and 2 cycles per year).

The first step in filling these gaps was to employ the discrete cosine transform (DCT) to obtain representative values from the DCT spectrum on either side of the two frequencies of primary interest. The mathematical steps required to calculate the DCT are given, for example, in Jain (1989). Means and standard deviations were calculated from these values and then used together with a random number generator to fill the gaps. Although the method is primarily objective, decisions must be made regarding how best to tune the filter and where in the DCT spectrum to obtain representative samples.

What should have been apparent from the outset is that there was no need to apply SSA to the data in order to remove the annual cycle. The DCT can serve not only as a gap filler but as a narrowband filter as well. The results of using the DCT as a narrowband filter are shown in Fig. A1. The annual cycle per se occurs at a relative frequency of 180 , which corresponds to 1 cycle per year (cpy), and the first harmonic at 360 or 2 cpy (Fig. A1a). By taking a closer look at the annual cycle (Fig. A1b), we observe a series of smaller oscillations that bracket the primary oscillation at $1 \mathrm{cpy}$ from relative frequencies of 170 to 190 that may correspond to the wellknown Gibbs phenomenon (e.g., Thomson and Emery 2014). Although this phenomenon is interesting in its own right, in the present case we are concerned with removing it since it is part and parcel of the annual cycle. Thus, we have removed the values from 167 to 193 . Similar steps were taken to remove the first harmonic at $2 \mathrm{cpy}$. At this point, as described above, spectral estimates from the DCT were taken from each side of the two primary maxima and used to fill the existing gaps. Last, although we have not done so, we could have used this procedure to produce confidence limits for the values that were used to fill the gaps as well.

\section{REFERENCES}

Abry, P., D. Veitch, and P. Flandrin, 1998: Long-range dependence: Revisiting aggregation with wavelets. J. Time Ser. Anal., 19, 253-266, https://doi.org/10.1111/1467-9892.00090.

Ackerman, B. W., 1981: Mesoscale Atmospheric Circulations. Academic Press, 495 pp.

Breaker, L. C., 2006: Nonlinear aspects of sea surface temperature in Monterey Bay. Prog. Oceanogr., 69, 61-89, https://doi.org/ 10.1016/j.pocean.2006.02.015.

— range persistence and scaling of sea surface temperature off the coast of California. J. Geophys. Res. Oceans, 124, 32063227, https://doi.org/10.1029/2018JC014922.

— , and E. M. Armstrong, and C. A. Endris, 2011: Establishing an objective basis for image compositing in satellite oceanography. Remote Sens. Environ., 114, 345-362, https://doi.org/ 10.1016/j.rse.2009.09.014.

Bunde, A., J. F. Eichner, S. Havlin, E. Koscielny-Bunde, H. J. Schellhuber, and D. Vyushin, 2004: Comment on "Scaling of atmosphere and ocean temperature correlations in observations and climate models." Phys. Rev. Lett., 92, 039801, https:// doi.org/10.1103/PhysRevLett.92.039801.

Capparelli, V., A. Vecchio, and V. Carbone, 2011: Long-range persistence of temperature records induced by long-term climatic phenomena. Phys. Rev. E, 84, 046103, https://doi.org/ 10.1103/PhysRevE.84.046103.

Checkley, D. M., and M. Lindegren, 2014: Sea surface temperature variability at the Scripps Institution of Oceanography Pier. J. Phys. Oceanogr., 44, 2877-2892, https://doi.org/10.1175/ JPO-D-13-0237.1.

Chen, Z., P. Ivanov, K. Hu, and H. Stanley, 2002: Effect of nonstationarities on detrended fluctuation analysis. Phys. Rev. E, 65, 041107, https://doi.org/10.1103/PhysRevE.65.041107.

Flandrin, P., P. Goncalves, and G. Rilling, 2004: Empirical mode decomposition as a filter bank. IEEE Signal Process. Lett., 11, 112-114, https://doi.org/10.1109/LSP.2003.821662.

,-- , and -2005 : EMD equivalent filter banks from interpretation to applications. The Hilbert-Huang Transform and its Applications, N. E. Huang and S. S. P. Shen, Eds., Interdisciplinary Mathematical Sciences, Vol. 5, World Scientific, 57-74.

Fraedrich, K., and R. Blender, 2003: Scaling of atmosphere and ocean temperature correlations in observations and climate models. Phys. Rev. Lett., 90, 108501, https://doi.org/10.1103/ physrevlett.90.108501.

Golyandina, N., V. Nekrutkin, and A. Zhigljavsky, 2001: Analysis of Time Series Structure: SSA and Related Techniques. Chapman and Hall/CRC, 305 pp. 
Hu, K., P. C. Ivanov, Z. Chen, P. Carpena, and H. E. Stanley, 2001: Effect of trends on detrended fluctuation analysis. Phys. Rev. E, 64, 011114, https://doi.org/10.1103/physreve.64.011114.

Huang, N. E., 2005a: Introduction to Hilbert-Huang transform and some recent developments. The Hilbert-Huang Transform in Engineering, N. E. Huang and N. A. Attoh-Okine, Eds., Taylor and Francis, 1-24.

- 2005b: Introduction to the Hilbert-Huang transform and its related mathematical problems. The Hilbert-Huang Transform and Its Applications, N. E. Huang and S. S. P. Shen, Eds., World Scientific, 1-26, 57-74.

— the. Hilbert spectrum for nonlinear and non-stationary time series analysis. Proc. Roy. Soc. London, 454A, 903-995, https://doi.org/10.1098/rspa.1998.0193.

Hurst, H. E., 1951: Long-term storage capacity of reservoirs. Trans. Amer. Soc. Civ. Eng., 116, 770-808.

Ihlen, E. A., 2012: Introduction to multifractal detrended fluctuation analysis in MATLAB. Front. Physiol., 3, 141, https:// doi.org/10.3389/fphys.2012.00141.

Jain, A. K., 1989: Fundamentals of Digital Image Processing. Prentice-Hall, Inc., 565 pp.

Jenkins, G. M., and D. G. Watts, 1968: Spectral Analysis and its Applications. Holden-Day, $525 \mathrm{pp}$.

Jones, S. F., and Y. Toba, 2001: Wind Stress over the Ocean. Cambridge University Press, 301 pp.

Kantelhardt, J. W., E. Koscielny-Bunde, H. H. A. Rego, S. Havlin, and A. Bunde, 2001: Detecting long-range correlations with detrended fluctuation analysis. Physica A, 295, 441-454, https://doi.org/10.1016/S0378-4371(01)00144-3.

—, S. A. Zschiegner, E. Koscielny-Bunde, S. Havlin, A. Bunde, and H. E. Stanley, 2002: Multifractal detrended fluctuation analysis of nonstationary time series. Physica A, 316, 87-114, https://doi.org/10.1016/S0378-4371(02)01383-3.

Luo, M., Y. Leung, Y. Zhou, and W. Zhang, 2015: Scaling behavior of global mean sea surface temperature anomalies. J. Climate, 28, 3122-3132, https://doi.org/10.1175/JCLI-D-13-00743.1.

Malamud, B. D., and D. L. Turcotte, 1999: Self-affine time series: Generation and analysis. Advances in Geophysics, Vol. 40, Academic Press, 1-90, https://doi.org/10.1016/S0065-2687(08) 60293-9.

Mandelbrot, B., and J. W. Van Ness, 1968: Fractional Brownian motions, fractional noises and applications. SIAM Rev., 10, 422-437, https://doi.org/10.1137/1010093.

Maraun, D., H. W. Rust, and J. Timmer, 2004: Tempting longmemory-On the interpretation of DFA results. Nonlinear Processes Geophys., 11, 495-503, https://doi.org/10.5194/npg11-495-2004.
Monetti, R. A., S. Havlin, and A. Bunde, 2003: Long-term persistence in the sea surface temperature fluctuations. Physica A, 320, 581-589, https://doi.org/10.1016/S0378-4371(02)01662-X.

Monin, A. S., V. M. Kamenkovich, and V. G. Kort, 1972: Variability of the Oceans. John Wiley and Sons, 241 pp.

Peng, C.-K., S. V. Buldyrev, S. Havlin, M. Simons, H. E. Stanley, and A. L. Goldberger, 1994: Mosaic organization of DNA nucleotides. Phys. Rev. E, 49, 1685-1689, https://doi.org/ 10.1103/physreve.49.1685.

Qian, X.-Y., W.-X. Zhou, and G.-F. Gu, 2009: Modified detrended fluctuation analysis based on empirical mode decomposition. arXiv, https://arxiv.org/abs/0907.3284.

Taqqu, M. S., V. Teverovsky, and W. Willinger, 1995: Estimators for long-range dependence: An empirical study. Fractals, 3, 785-798, https://doi.org/10.1142/S0218348X95000692.

Thomson, D. J., 1982: Spectrum estimation and harmonic analysis. IEEE Proc., 70, 1055-1096, https://doi.org/10.1109/ PROC.1982.12433.

Thomson, R. E., and W. J. Emery, 2014: Data Analysis Methods in Physical Oceanography. 3rd ed. Elsevier, 728 pp., https:// doi.org/10.2307/1353059.

Tsonis, A. A., P. J. Roebber, and J. B. Elsner, 1999: Long-range correlations in the extratropical atmospheric circulation: Origins and implications. J. Climate, 12, 1534-1541, https:// doi.org/10.1175/1520-0442(1999)012<1534:LRCITE>2.0.CO;2.

,-- , and — 2000: On the existence of spatially uniform scaling laws in the climate system. Paradigms of Complexity, M. Novak, Ed., World Scientific, 25-28.

Vyushin, D. I., P. J. Kushner, and J. Mayer, 2009: On the origins of temporal power-law behavior in the global atmospheric circulation. Geophys. Res. Lett., 36, L14706, https://doi.org/ 10.1029/2009GL038771.

Watson, S., 2014: Quantifying the variability of wind energy. Wiley Interdiscip. Rev.: Energy Environ., 3, 323-407, https://doi.org/ 10.1002/wene.95.

Wells, N. C., 2012: The Atmosphere and the Ocean. John Wiley and Sons, 411 pages.

Witt, A., and B. D. Malamud, 2013: Quantification of long-range persistence in geophysical time series: Conventional and benchmark improvement techniques. Surv. Geophys., 34, 541651, https://doi.org/10.1007/s10712-012-9217-8.

Wu, Z., and N. E. Huang, 2008: Ensemble empirical mode decomposition: A noise-assisted data analysis method. Adv. Adapt. Data Anal., 1, 1-41, https://doi.org/10.1142/S1793536909000047.

Yashayaev, I. M., and I. I. Zveryaev, 2001: Climate of the seasonal cycle in the North Pacific and the North Atlantic Oceans. Int. J. Climatol., 21, 401-417, https://doi.org/10.1002/ joc.585. 